aetiology is primarily PPHN. This is supported by the paper which demonstrates a reduction in Nitric Oxide Synthetase following PROM.

We suggest that, where possible, mothers with PROM and oligohydramnios should be delivered in a tertiary referral centre where iNO therapy is immediately available.

\section{G134(P) QUESTIONNAIRE BASED NATIONAL SURVEYS - THE HURDLES AND HEART ACHES!}

${ }^{1}$ A Lakshmanan, ${ }^{1} \mathrm{~T}$ Crosby, ${ }^{1} \mathrm{~L}$ Lee, ${ }^{2} \mathrm{~W}$ Kelsall. 'Department of Neonatal Medicine, Nottingham University Hospital NHS Trust, Nottingham, UK; ${ }^{2}$ Department of Neonatal Medicine, Addenbrookes Hospital, Cambridge, UK

10.1136/archdischild-2015-308599.132

Introduction British Paediatric Surveillance Unit (BPSU) promotes study of rare diseases and infections by orange card reporting system and subsequent questionnaire based surveillance. We conducted a study of surgically ligated PDA in preterm babies between September 2012 and September 2013. The questionnaire had 10 parts with a total of 43 questions to answer. 18 of these were generic questions and could have been answered by non-medical staff. The questionnaire was designed after balancing the number of questions against the completeness of the data required. The aim of this abstract is to outline some of the difficulties of such questionnaire based national surveys and possible solutions.

Results 531 cases were reported and questionnaires posted to the relevant Consultants. $10 \%$ of these questionnaires were not returned and similar percentages were reported in error (see Figure 1).

Multiple reporting occured in 96 cases - 83 of them were duplicates, 12 were triplicates and 1 case was reported by 4 different clinicians. Incomplete data was provided in 96 cases. This was less with multiple reporting ones 11/96 (11.4\%) compared to $82 / 199(41 \%)$ cases which were reported once. Multiple reporting thus helped us to obtain more information about the case. But this also resulted in some discrepancies in the reporting of $12(12.5 \%)$ cases.

Conclusions In our experience, we felt that email correspondence was quicker and had a better response rate compared to paper correspondence. Division of the questionnaire into medical and non-medical parts could enable the clinician to concentrate on the relevant medical information and leaving the nonmedical information to be filled in by administrative staff.

The longer the time that lapsed between the case being reported to the BPSU and the questionnaire being completed by the Consultant, the more incomplete was the reported data.

Improvements in data ascertainment might be possible in a neonatal project if the research team were able to access national

\section{Total Questionnaires sent $\mathbf{=} \mathbf{5 3 1}$}
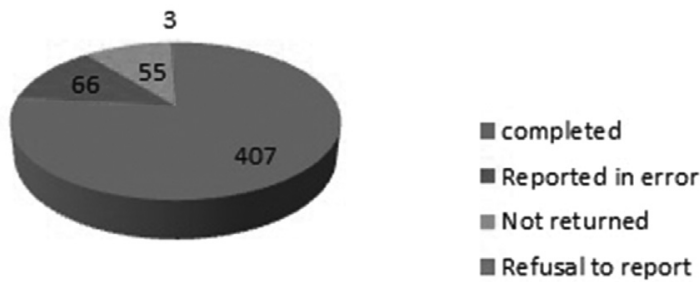

Abstract G134(P) Figure 1 databases such as BADGER system for named patients to improve the accuracy and quality of data.

This type of surveillance has great epidemiological and clinical impact. Identifying key issues and addressing them early can ensure high quality data is collected and disseminated.

\section{G135(P) CHASING THE WELSH DRAGON: A REVIEW OF THE OUTCOME OF INFANTS WITH NEONATAL ABSTINENCE SYNDROME OVER A 10 YEAR PERIOD}

IJ Joy, ${ }^{2}$ A Allman. 'Paediatrics, Cwm Taf NHS Trust, Pontyclun, UK; ${ }^{2}$ Neonatology, Aneurin Bevan Health Board, Newport, UK

\subsection{6/archdischild-2015-308599.133}

Introduction Neonatal abstinence syndrome (NAS) is a complex, multi system disorder which not only involves acute withdrawal, but also long term morbidities. We looked at infants with NAS at the Royal Gwent Hospital (RGH), and studied the impact of the condition on their health over a 10 year period.

Methods Infants born in RGH with a diagnosis of NAS between 2000 - 2010 were identified from the neonatal and transitional care admission registers. Clinical work station documents plus the neonatal database were used to gather information.

Results The total number of infants with a diagnosis of NAS was $231 ; 101$ were admitted to the neonatal unit. $83 \%$ of babies had urine toxicology performed and the commonest result was methadone plus opiates (26\%). Of the babies admitted to the unit, the average duration of stay was 20 days and all required pharmacological management. In our cohort, 15\% developed ophthalmological disorders, including squint, nystagmus and delayed visual maturation; $13 \%$ had developmental problems and $44 \%$ of these showed speech and language delay. $43 \%$ of the 231 infants attended A\&E at least once during early childhood; head injury was common.

Conclusion The incidence of NAS inSouth Gwentin the decade 2000 to 2010 is around 8/1000 live births. Approximately 1 in 7 infants develop ophthalmological disorders and 1 in 8 have developmental problems. We conclude that infants with NAS in the neonatal period have significant ongoing morbidity and remain a vulnerable group who may benefit from enhanced targeted health surveillance.

\section{G136(P) A 4 YEARS REVIEW OF CONJUGATED HYPERBILIRUBINAEMIA IN A TERTIARY NEONATAL REFERRAL CENTRE}

R Alia, H Shore, A Turi. Neonatology, Leeds Teaching Hospitals NHS Trust, Leeds, UK

\subsection{6/archdischild-2015-308599.134}

Aim To establish the incidence of conjugated hyperbilirubinaemia over a 4 years period in a tertiary neonatal unit and to review the demographic data, the investigations and treatments they received.

Method A retrospective observational study between 01/06/ 2010 and 28/02/2014. Data was retrieved from the neonatal database BADGER. Search term: conjugated hyperbilirubinaemia or ursodeoxycholic acid.

Result Less than 1\% (40/5237) admissions to the neonatal unit had conjugated hyperbilirubinaemia. 70\% were less than 33 weeks gestation. 60\% weighed less than 1251g. 33 infants (82\%) received total parenteral nutrition (TPN). Variable numbers of babies were investigated for different conditions ( 24 infants had 
TORCH screen, 15 infants had a1 antitrypsin level, 13 infants had Gal-1-p level, 25 infants had abdominal ultrasound scan and 26 infants had urine metabolic screen). Regarding diagnosis: 18 babies had TPN related cholestasis, 3/40 had biliary atresia, one had choledochal cyst. 3/40 had liver failure. All babies received ursodeoxycholic acid and 80-90\% had vitamin supplements (vitamin K, E, and/or dalavit). 27 babies were referred to the liver team.

Conclusion Conjugated hyperbilirubinaemia is rare on neonatal units. Infants who are less than 33 weeks gestation and less than $1251 \mathrm{~g}$ are most at risk. The majority were diagnosed with TPNassociated cholestasis. It is important to develop guidance for diagnosis and treatment for conjugated hyperbilirubinaemia in the neonate to ensure cost effectiveness of a more streamlined investigation plan, limiting discomfort and risk of anaemia for babies and also consistency in management.

\section{G137(P) PATENT DUCTUS ARTERIOSUS OF PREMATURITY: TO TREAT OR NOT TO TREAT?}

VM Shetthalli, S Nittur, A Wong, O Uzun. Department of Paediatric Cardiology, University Hospital of Wales, Cardiff, UK

\subsection{6/archdischild-2015-308599.135}

Background Incidence of patent ductus arteriosus in neonates is $30-60 \%$ depending on the degree of prematurity. Although patent ductus arteriosus is associated with significant morbidity and mortality in preterm infants, there is a wide variation in management of patent ductus arteriosus in preterm infants.

Methods We reviewed of our 22 year experience from 1992 to 2013 in all neonates diagnosed with a haemodynamically significant patent ductus arteriosus at the University Hospital of Wales. Haemodynamically significant patent ductus arteriosus is described as: (1) left atrium/aortic ratio of $>1.5$, (2) significant left heart volume overloading, (3) bounding femoral pulses, (4) hyperactive precordium and (5) persistent ventilator requirements.

Results A total of 200 cases were eligible. The results are summarised in the Table 1:

\begin{tabular}{lll} 
Abstract G137(P) Table 1 & & \\
\hline & Pharmacologically treated & Not treated \\
\hline $\mathrm{n}$ & $88(44 \%)$ & $112(56 \%)$ \\
Males n (\%) & $42(47 \%)$ & $65(57 \%)$ \\
Median gestation in weeks & 26 & 27 \\
(Q3-Q1) & $(25-28)$ & $(26-31)$ \\
Median Birth weight in grams & 850 & 1020 \\
(Q3-Q1) & $(700-1077)^{*}$ & $(820-1387)^{*}$ \\
Median NICU stay & 62 days & 34 days \\
(Q3-Q1) & $(27-104.75)^{*}$ & $(13.5-64)^{*}$ \\
Median left atrium/aortic ratio & 1.72 & 1.43 \\
(Q3-Q1) & $(1.5-2)^{*}$ & $(0.9-2)^{*}$ \\
Treatment Success rate & $37(42 \%)$ & $\mathrm{N} / \mathrm{A}$ \\
Surgery/catheter & $36(40.9 \%)^{*}$ & $21(18.7 \%)^{*}$ \\
Necrotising Enterocolitis (NEC) & $14 / 88(15.9 \%)$ & $20 / 112(17.8 \%)$ \\
Intraventricular Haemorrhage (IVH) & $12 / 38(13.6 \%)$ & $13 / 48(11.5 \%)$ \\
Chronic Lung Disease (CLD) & $29 / 38(76.13 \%)$ & $29 / 48(60.4 \%)$ \\
Death & $12 / 88(13.6 \%)$ & $13 / 88(11.6 \%)$ \\
\hline *P = < 0.05. & & \\
(Q3-Q1) = Interquartile range. & & \\
& &
\end{tabular}

Pharmacological treatment $=$ Treatment with either Indomethacin or Ibuprofen
Conclusion There were no significant differences in NEC, IVH, CLD or death between treatment and no-treatment group despite the former cases being at lower gestations with lower birth weights. Our result may lend further support towards nonsurgical management of patent ductus arteriosus with medication if treatment is not considered to be contraindicated.

\section{G138(P) THE RAPID DEBRIEF: A TOOL THAT TRANSFORMS LEARNING AND SYSTEM CHANGE}

${ }^{1} \mathrm{R}$ Puttha, ${ }^{1} \mathrm{~S}$ Yuen, ${ }^{2} \mathrm{R}$ Thalava, ${ }^{1} \mathrm{H}$ Abdalla, ${ }^{1} \mathrm{~N}$ Moghal. ${ }^{1}$ Paediatrics, George Eliot Hospital, Nuneaton, UK; ${ }^{2}$ Orthopaedics, Tameside General Hospital, Manchester, UK

\subsection{6/archdischild-2015-308599.136}

Aim To test the rapid debrief tool as a way of extracting immediate learning to implement system changes following the care of the critically child; overcoming the dispersion of people and memories in the traditional incident reporting cycles.

Method A rapid debrief was tested immediately after the care of a critically ill child was completed by the team. A template was used to collect what improvements were needed technically (resources, skills) as well human factors such as communication and leadership. Action plans were generated by the team. The debrief and action plan was then circulated to all staff and discussed at the weekly service meetings. Outcomes were monitored by the Paediatric Resuscitation Group.

Results A total of 29 rapid debriefs were completed over 12 months, generating 81 action plans, of which 50 have been completed. Many of the actions were completed before the incident forms reached the clinical governance system. 20 related to equipment, 5 to medications, 7 to team issues (communication, leadership), 10 training issues and 11 planning and organisation wide issues. Compared to the year previous to the rapid debrief, clinical Incident reporting now shows a 1.7 times increase of low risk incidence reporting; incidents of moderate or high risk have been reduced by half. Staff feedback has been very positive. The learning outcomes include the development of safe handover tools, improving resuscitation resources and team needs, incorporating human factors into the resuscitation training to build team resilience and an open challenging culture.

Conclusion The rapid debrief has helped improve our care of the critically ill child through the immediate extraction of learning and implementation of improvements. The tool enables faster system change compared to traditional reporting governance systems.

\section{G139(P) ADVANCED NEONATAL EMERGENCIES: CONFIDENCE AND EXPERIENCE LEVELS OF NEW REGISTRARS}

JM Parasuraman. Paediatrics, Severn Deanery, Taunton, Somerset, UK

\subsection{6/archdischild-2015-308599.137}

Aims Paediatric trainees, who step up to the role of registrars at ST4/5 levels, are required to be competent in neonatal emergencies. Some of the competencies as highlighted by the RCPCH curriculum for level 2 are:

1. Ability to lead initial and advanced resuscitation when required

2. Ability to intubate pre-term babies without supervision

3. Understanding of the principles and ability to initiate mechanical ventilation 